\title{
Immunosensor Using Surface Plasmon Resonance for C-Reactive Protein Detection
}

\author{
Edmar CASA, ${ }^{\text {a b, c }}$ Chikako Kurosawa, , d Shigeru Kurosawa, ${ }^{\text {a }}$ Hidenobu Aizawa, ${ }^{a}$ \\ Jong-Won PARK, and Hiroaki SUZUKI ${ }^{\mathrm{d}}$
}

\author{
aNational Institute of Advanced Industrial Science and Technology (AIST) (1-1-1 Higashi, Tsukuba, Ibaraki 305- \\ 8565, Japan) \\ bJapan International Cooperation Agency (JICA) (3-6 Koyadai, Tsukuba 305-0074, Japan) \\ 'Industrial Technology Development Institute (ITDI) (Bicutan, Taguig, 1631 Metro Manila, Philippines) \\ ${ }^{d}$ University of Tsukuba (1-1-1 Tennoudai, Tsukuba, Ibaraki 305-8573, Japan)
}

Received August 31, 2005 ; Accepted November 18, 2005

\begin{abstract}
An immunosensor for human C-reactive protein (CRP) detection was developed by combining a portable surface plasmon resonance (SPR) machine with an anti-human C-reactive protein monoclonal antibody (AbCRP)-immobilized gold surface of the microfluidic SPR sensor chip (MSSC). The gold surface of MSSC was chemically modified using self-assembly monolayer technique with 4,4'-dithiodibutyric acid. Subsequently, AbCRP was immobilized on the chemically-activated gold surface of the MSSC by chemical adsorption and CRP determinations were performed using immunoreaction. The detection limit for CRP concentration by this developed SPR method was 1.0 $\mu \mathrm{g} / \mathrm{ml}$.
\end{abstract}

Key Words : Immunosensor, Surface Plasmon Resonance, Microfluidic Sensor Chip, C-Reactive Protein

\section{Introduction}

Surface plasmon resonance (SPR) technique continues to gain recognition as a powerful tool for investigating biological interactions because of its speed of detection, high specificity, high sensitivity, non-labeling analysis of biomolecular interactions, and the possibility of on-line real time analysis. ${ }^{1,2)}$ A common principle of the SPR immunosensor is that the "detector molecules" are immobilized on the gold surface of SPR sensor chips in such a way that a specific signal is obtained from the sensor when the detector molecules react selectively with the biomolecules that they are designed to detect. That phenomenon is based on the fact that if the surface is modified by binding, then the optical properties will change, particularly the refractive index of the environment that is affected by the binding. That change, which is very accurately measurable, can then be used to detect both the binding rate and its extent. When the incident light strikes the back of a thin gold layer, an evanescent field is generated the interface of the glass slide and the metal film, inducing the generation of a surface plasmon from the free electrons of the metal film. ${ }^{3}$ ) To date, SPR has been used to measure protein adsorption on polymer surfaces, protein binding to DNA, analysis of protein arrays, and antibody-antigen interactions. A variety of surface-based detection principles are employed for SPR immunosensor studies. ${ }^{4-9)}$

A highly characteristic acute-phase protein that is currently suggested as a marker of inflammation along with serum cholesterol is C-reactive protein (CRP). It has been used as a clinical tool for monitoring autoimmune and infectious diseases such as rheumatoid arthritis and development and progression of atherosclerosis. ${ }^{10}$
Hepatic synthesis of CRP dramatically increases its plasma concentration from a normal level of $<1 \mu \mathrm{g} / \mathrm{ml}$ to $100-500 \mu \mathrm{g} / \mathrm{ml}$ following acute tissue damage or during the course of infectious and non-infectious diseases. The conventional sensor device which measures CRP concentration in the serum rapidly should be necessary. But, the sensor device hasn't been developed yet. Thus, we constructed an conventional flow-type immunosensor system to combine a microfluidic SPR sensor chip with immobilized antibody for immunoreaction on chip. This work describes the development of SPR immunosensors for CRP detection by combining a portable SPR machine and a microfluidic sensor chip with immobilized antibody.

\section{Experimental}

4,4'-Dithiodibutyric acid (DDA), N-hydroxysuccinimide (NHS), 1-ethyl-3-3-dimethylaminopropylcarbodiimide, hydrochloride (EDC), bovine serum albumin (BSA), $\gamma$ globulin, and glycine were used as received. Anti-human CRP monoclonal antibody (AbCRP), recombinant human C-reactive protein (CRP), and CRP-free human pooled serum were purchased from Oriental Yeast Co. Ltd., Japan. Solutions of antibody, blockers, and antigen were prepared with PBS. All solutions were prepared using ultrapure water $(18 \mathrm{M} \Omega / \mathrm{cm})$ obtained from Milli-Q ultrapure water system. The SPR system used in this study comprised mainly an SPR analyzer (NTT Advanced Technology Corp., Japan), the SPR sensor chip, a syringe pump, and an injector with a sample loop. To compare the amount of sensor responses for antibody immobilization and immunoreaction, we designed and fabricated two types of microfluidic sensor chip with same channel 
depth $(10 \mu \mathrm{m})$ and different configurations (circular and rectangular). Thin gold-film sputtered glass plates served as a base for SPR sensor chip whereas a chemically-inert and easily moldable PDMS thin film (locally fabricated) having microchannel structures acted as flow cells (Table 1). In a typical SPR experiment, the incident light at which the intensity of the reflected light is at minimum, called the "SPR angle" was monitored using a computer. A schematic diagram of the SPR sensor set-up is shown in Fig. 1. Analyte solutions were allowed to flow at a rate of $10 \mu \mathrm{l} / \mathrm{min}$, and the phosphate buffered saline (PBS, pH 7.4) was the carrier solution. The injected volume of the analyte was $20 \mu$ l. All procedures were performed at $20 \pm 0.1{ }^{\circ} \mathrm{C}$, unless otherwise specified.

Gold $(\mathrm{Au})$ was sputtered on a glass cover plate $(16 \times$ $16 \times 0.15 \mathrm{~mm}$ ) using a thin-film process. Chromium acts as an adhesion layer and was first sputtered to the bare glass cover plate at $c a$. 5 -nm-thickness. Subsequently, Au was deposited (by sputtering) over the chromium layer to a thickness of $c a$. $33 \mathrm{~nm}$. Thickness of the metal depositions was measured using a spectroscopic ellipsometer. Two types of poly(dimethylsiloxane) (PDMS) microfluidic flow cells were fabricated using photolithography method. ${ }^{11,12)}$ Chemically inert and easily moldable PDMS thin films having microchannel structure acted as flow cells for the SPR sensor chips. A glass substrate with patterned ultra-thick photoresist (SU-8) was used as a mold master. After patterning, the pre-polymer of PDMS is poured onto the mold master and baked (or cured) at $100^{\circ} \mathrm{C}$. The cured PDMS, when peeled off from the mold master, becomes ready for use.

Table 1 Properties of different types of microfluidic flow cell used in this study.

\begin{tabular}{ccc}
\hline Type & $\begin{array}{c}\text { Microfluidic Type A } \\
\text { (circular) }\end{array}$ & $\begin{array}{c}\text { Microfluidic Type B } \\
\text { (rectangular) }\end{array}$ \\
\hline Shape & $\mid \mathrm{J}$ & $10 \mu \mathrm{m}$ \\
Channel depth & $10 \mu \mathrm{m}$ & $0.120 \mu \mathrm{L}$ \\
Volume capacity & $0.377 \mu \mathrm{L}$ & \\
\hline
\end{tabular}

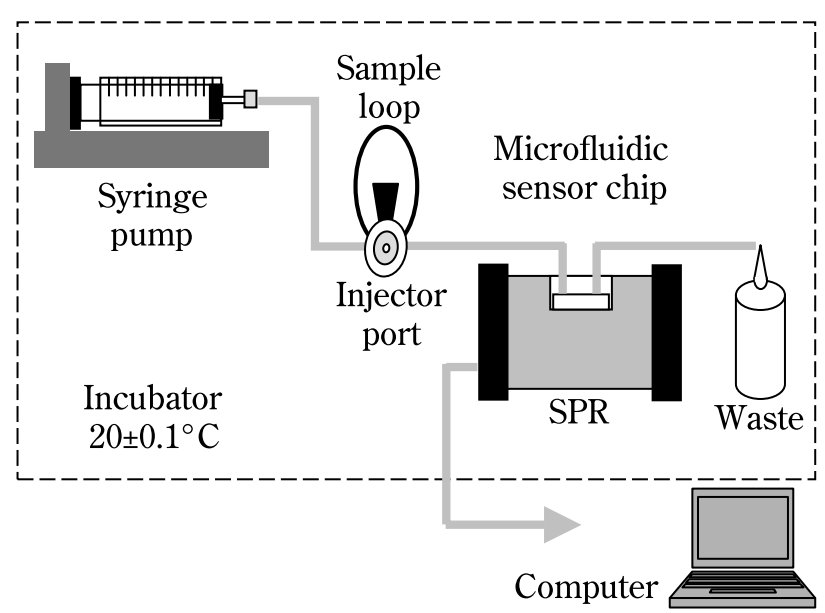

Fig. 1 Schematic diagram of the SPR sensor set-up.
Surface modification method based on molecular selfassembly is used to form a sensing film on the gold surface. The gold surface of the SPR sensor chip was placed in $1.0 \mathrm{mM}$ of DDA ethanolic solution and shaken for 30 min (to form S-Au binding from the disulfide bond of DDA), followed by rinsing with ethanol and drying under nitrogen gas. The gold surface of the sensor chip was then immersed in a solution containing NHS and EDC to activate the ester group on the gold surface. It was then allowed to react for $10 \mathrm{~min}$. Then, $10 \mathrm{ml}$ of water was added to the reaction mixture, and allowed to react further for another $5 \mathrm{~min}$. The surface was rinsed with water and dried under nitrogen gas. The activated gold sensor chip was attached to the microfluidic flow cell made from PDMS with the gold film exposed to the solution. The test solutions were circulated at a fixed flow rate. Immobilization of $\gamma$-globulin or AbCRP was done on the sensor chip by chemical adsorption. AbCRP was injected, followed by $20 \mathrm{mM}$ glycine to block the unreacted activated esters of the monolayer. A PBS solution containing $0.1 \%$ BSA was used to block the non-specific binding sites. Different concentrations of antigens (CRP) were then injected and allowed to flow into cell for $25 \mathrm{~min}$. The difference in SPR angles before the immobilization and after the final PBS wash corresponds to the amount of immobilized analyte.

\section{Results and Discussion}

Preliminary experiments were undertaken to determine the suitable type of flow cell and the optimum amount of immobilized molecule to the biorecognition surface formed on a chemically-modified gold layer of the sensor chip to be used for the succeeding immunoreaction study. Table 1 lists the properties of different flow cells used in this study. To select a suitable sensor chip with high sensitivity, different concentrations of $\gamma$-globulin solutions were flowed into each flow cell; then the SPR angle changes were detected. Results showed that the SPR angle shift of microfluidic Type B flow cell is greater than that of the Type A flow cell (data not shown). It was further observed that the SPR angle change for Type B flow cell indicated higher signal shift than Type A at lower concentration ranges of $\gamma$-globulin ( $<150 \mu \mathrm{g} / \mathrm{ml})$, but had almost the same SPR response at a higher concentration. This might be because, although both microfluidic flow cells have the same channel depth. The volume capacities and the configuration (shape) differ significantly. The flow path of the low concentration analyte using Type A flow cell might be quite dispersed throughout the surface. Alternatively, some might not pass through the point of the detection of the SPR. However, the Type B flow cell has a narrower configuration, so the analyte is able to pass all throughout the SPR detection point. We selected the Type B flow cell for SPR experiments.

The biorecognition surface of the gold surface of the SPR sensor was treated to immobilize the antibody using DDA with NHS/EDC. The antibody is immobilized through covalent binding between the amino groups of the antibody and the active ester groups of the activated 


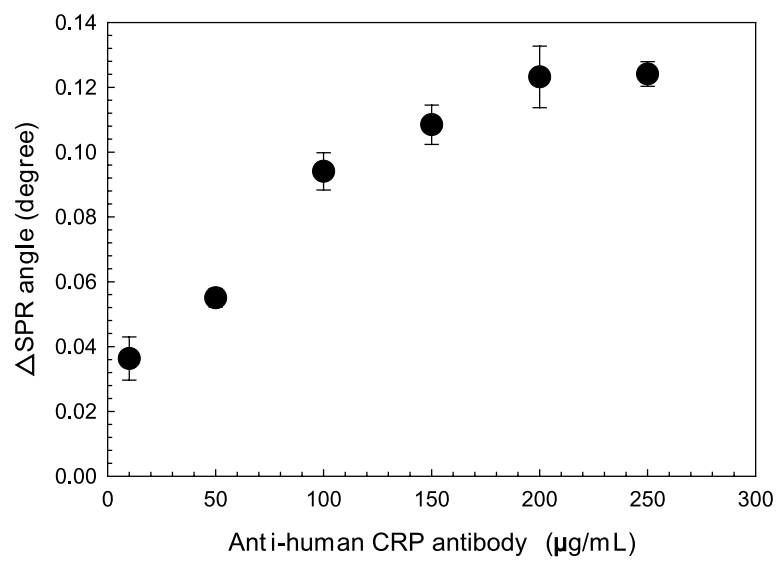

Fig. 2 Dose response of an anti-human CRP antibody on a chemically-activated gold sensor-chip surface.

gold surface. The adsorption curve of AbCRP on the sensor surface was determined (Fig. 2). The maximum amount of AbCRP adsorbed on the chemically-activated gold sensor chip surface was $200 \mu \mathrm{g} / \mathrm{ml}$. We assumed the monolayer adsorption manner of AbCRP molecular on the chemically-activated gold sensor chip surface over this antibody concentration. This amount confirms the results with $\gamma$-globulin in the preliminary study.

Figure 3 shows the dose response of immunoreaction between CRP and AbCRP immobilized at $200 \mu \mathrm{g} / \mathrm{ml}$. The graph showed a sigmoidal pattern response. The lowest detection limit for CRP using direct immunoreaction was $1.0 \mu \mathrm{g} / \mathrm{ml}$, which corresponds to an SPR angle change of $3.79 \times 10^{-3} \mathrm{deg}$. The binding of CRP to the immobilized AbCRP was saturated after $10 \mu \mathrm{g} / \mathrm{ml}$. The detection range of the SPR immunosensor for CRP is within $10 \mathrm{~min}$. It is noteworthy that the normal concentration of CRP in the human body is less than $1 \mu \mathrm{g} / \mathrm{ml}$. This result demonstrates that SPR immunoassay described here is quite capable of detecting inflammatory and infectious processes that occur in the human body.

Selectivity of the sensor chip for C-reactive protein was also produced by injecting a 1.0\% CRP-free human serum PBS mixed with $10 \mu \mathrm{g} / \mathrm{ml} \mathrm{CRP} \mathrm{into} \mathrm{the} \mathrm{flow} \mathrm{cell}$ after AbCRP was immobilized. The SPR response is identical to that of CRP alone, indicating that other components of the human serum did not interfere with the determination of CRP ( $\triangle \mathrm{SPR}$ angle was obtained 0.036 degree). Further investigations will be carried out to determine the applicability of the developed SPR immunosensor through analyses using real samples.

\section{Acknowledgements}

This work was supported financially in part by a Grant-in-Aid for Scientific Research on Priority Areas

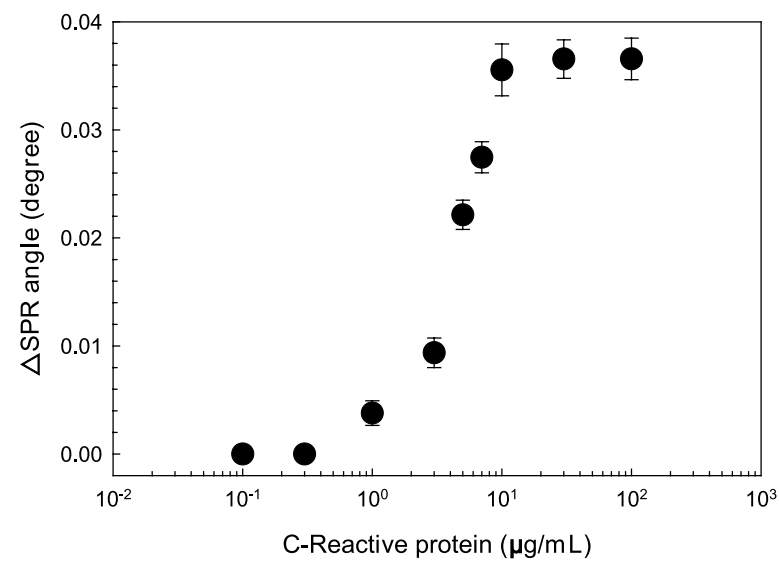

Fig. 3 Dose response of immunoreaction between the CRP and an anti-CRP antibody immobilized sensor chip.

(16040217) from MEXT, for “Application of permselective and biocompatible membranes for the improvement quartz crystal microbalance biosensors" from the Daiwa Anglo-Japanese Foundation, and for "Study on monitoring of environmental risk compounds such as dioxins and endocrine disruptors using sensing systems" from the Ministry of the Environment, Japan.

\section{References}

1) C. L. Baird and D. G. Myszka, J. Mol. Recognit., 14, 261 (2001).

2) G. B. Sigal, M. Mrksich, and G. M. Whitesides, J. Am. Chem. Soc., 120, 3464 (1998).

3) L. G. Fägerstam, Ȧ. F. Karlsson, R. Karlsson, B. Persson, and I. Rönnberg, J. Chromatography, 597, 397 (1992).

4) S. Toyama, N. Doumae, A. Shoji, and Y. Ikariyama, Sensors \& Actuators B, 65, 32 (2000).

5) T. Nishimura, E. Hifumi, T. Fujii, Y. Niimi, N. Egashira, K. Shimizu, and T. Uda, Electrochemistry, 68, 916 (2000).

6) S. Sonezaki, S. Yagi, E. Ogawa, and A. Kondo, J. Immunological Methods, 238, 99 (2000).

7) M. Shimomura, Y. Nomura, W. Zhang, M. Sakino, K.-H. Lee, K. Ikebukuro, and I. Karube, Anal. Chim. Acta, 434, 223 (2001).

8) M. Suzuki, F. Ozawa, S. Wakako, and S. Aso, Anal. Bioanal. Chem., 372, 301 (2002).

9) D. R. Shankaran, K. V. Gobi, T. Sakai, K. Matsumoto, K. Toko, and N. Miura, Biosens. Bioelectron., 20, 1750 (2005).

10) W. Schramm, T. Yang, M. E. Meyerhoff, and L. G. Bachas, Clin. Chem. 36, 1360 (1990).

11) H. Suzuki and R. Yoneyama, Sensors and Actuators B, 96, 38 (2003).

12) H. Suzuki and Y. Matsugi, Sensors and Actuators B, 98, 101 (2004). 